\title{
Crisis económica, políticas, desempleo y salud (mental).
}

\author{
Economic crisis, politics, unemployment and (mental) health.
}

\author{
Antonio Espino Granado ${ }^{\text {. }}$
}

${ }^{a}$ Médico Psiquiatra.

Correspondencia: Antonio Espino Granado (to.pino@telefonica.net)

RESUMEN: En la ponencia del último Congreso de la AEN "Vida laboral, estrés y salud (mental)" se analizaron los problemas de estrés laboral que estaban llegando de manera creciente a nuestras consultas generando distintos problemas emocionales sobre los que tratamos de dilucidar su impacto psicopatológico y clínico real.

Con el desarrollo de la crisis financiera en nuestro país ha cogido protagonismo una forma severa de estrés relacionada con la ausencia de trabajo contra la voluntad del trabajador sin expectativas de solución en el corto plazo. Y su doble, la precariedad laboral, el trabajo sin derechos. Estas formas crecientes de estrés, más frecuentes en varones, pueden trascender los cuadros ansioso-depresivos habituales y dar lugar a una clínica psiquiátrica más severa con riesgo para la propia vida.

Analizar este problema es el objetivo del artículo, que desarrolla las interrelaciones entre la gestión de la crisis de la deuda, el alto desempleo mantenido y su impacto negativo sobre la salud (mental), con especial hincapié en la patología psíquica generada, la mortalidad general y el suicidio, los cambios en la demanda sanitaria y en la capacidad y organización de los sistemas sanitarios frente a la misma y las medidas dirigidas a mitigar su impacto negativo sobre la salud (mental).

El autor del artículo se pregunta qué razones existen para que un problema de tanta magnitud siga teniendo tan poca repercusión investigadora en nuestro país.

PALABRAS CLAVE: Crisis financiera. Desempleo. Precariedad laboral. Recortes de la protección sanitaria. Trastornos adaptativos. Depresión. Suicidio.
ABSTRACT: During the last Congress of AEN (The Spanish Association of Neuropsiquiatry) the paper "Working Life, Stress and (Mental) Health" analyzed the increasing situations of labor stress that were coming to our consultation. At that moment we tried to explain the psychopathological and real clinical impact of these emotional problems.

As the financial crisis has advanced in our country, a particularly malignant form of labor stress has taken prominence: joblessness. This phenomenon is against the worker's will and does not enable short-term solutions. Other forms of malignant labor stress are job insecurity and employers' lack of rights. These increasing types of stress - which are more frequent in men - can transcend the regular anxiety-depressive disorders causing severe psychiatric symptoms dangerous to life.

The aim of this article is to analyze in depth this problematic. It expound on the connection between the management of the debt crisis, the long-term unemployment and its negative impact on (mental) health. An emphasis is made on the psychiatric pathology generated, the global mortality and suicide, the changes in health services' demand and the system ability and organization to face it, together with the measures that could be taken to mitigate its negative impact on people's (mental) health.

The author wonders what are the reasons why a problem of this magnitude has such a small research impact in Spain.

KEY WORDS: Financial Crisis. Unemployment. Work Precariousness. Cuts Health Care. Adjustment Disorders. Depression. Suicide. 
«Guardaos de los falsos profetas, que disfrazados de ovejas, son lobos rapaces.

Por sus frutos los conoceréis".

Del Evangelio según Mateo

\section{UNO. El trabajo en nuestra sociedad}

¿Qué significa hoy "el trabajo”?

El trabajo (remunerado) ha ocupado históricamente un papel central en la vida de las personas.

Más allá de la cobertura económica garante de la subsistencia propia y de la familia, proporciona un conjunto de "nutrientes" necesarios para nuestra existencia y que sentimos especialmente cuando los perdemos: introduce orden en nuestra actividad cotidiana, facilita la organización estructurada y estructurante de nuestra vida, aporta relaciones sociales más allá del ámbito familiar, amplía nuestras metas y objetivos personales, participa en la definición de nuestro estatus social, nos aporta identidad personal frente a los demás y mantiene y fomenta nuestra vida activa al imponernos la realización concertada de una actividad, algo esencial si tenemos en cuenta que el mantenerse activo y ocupado es una de las principales motivaciones para trabajar y que uno de los mayores costes psicológicos de estar desempleado es, precisamente, el derivado de la propia inactividad (1).

Sin embargo, los objetivos de pleno empleo que caracterizaron a las sociedades de posguerra se han ido diluyendo a partir de la crisis de los años 70 (2).

La globalización financiera y la deslocalización de la producción de bienes de consumo ha aumentado la independencia de las élites económicas frente al mundo del trabajo. La actual primacía del mercado (global) sobre los territorios (Estados) influye de forma negativa sobre el grado de autonomía y libertad real con que cuentan las personas para desarrollar su proyecto vital (3).

El déficit de empleo disponible se ha convertido en condición estructural creciente en las nuevas sociedades capitalistas "de mercado", agravado en la actualidad por la recesión económica y la ineficacia de gran parte de las medidas tomadas frente a la misma por los gobiernos.

\section{La vida laboral de las personas}

A lo largo de su vida las personas van construyendo su "vida profesional", la suma de la experiencia del primer empleo (remunerado), las distintas condiciones de desempeño (grado de estabilidad, de reconocimiento y de regulación del mis- 
PARA EL DEBATE

mo), su adecuación a las competencias profesionales, los cambios de trabajo, las mejoras en el mismo, su pérdida y las condiciones de desempleo; su duración y la experiencia de buscar uno nuevo; la calidad y adecuación del nuevo empleo y por último, el impacto de los trabajos sumergidos y no regulados.

Las distintas situaciones de nuestra vida laboral afectan a nuestro mundo emocional e influyen sobre nuestra salud (mental): no es lo mismo disponer de un trabajo fijo que eventual, bien o mal remunerado, con o sin derechos laborales, adecuado a nuestras competencias profesionales o no.

Situaciones como la edad (jóvenes, mediana edad), el género (hombre/mujer), la duración del tiempo de desempleo (corto/ largo), el grado de apoyo social (institucional y comunitario), el grado de implicación previa con el trabajo o la variación en los ingresos económicos resultante de su pérdida, también influyen sobre el impacto emocional de las diferentes condiciones laborales $(4,5)$.

Sobre el impacto diferencial del trabajo - y su ausencia -, según género y grupos de edad.

Los desempleados constituyen un grupo cada vez menos homogéneo en la sociedad actual (6). La experiencia del desempleo (y el valor del empleo remunerado) varía según sea la situación personal, el momento laboral y el contexto cultural, habiéndose estudiado características especiales en el caso de los jóvenes (“diferencias por grupos de edad") y de la mujer ("diferencias de género").

- Importancia del primer empleo

La experiencia de la búsqueda del primer trabajo y el compromiso ejercido hacia el mismo influye sobre el propio proceso de socialización laboral en los jóvenes.

Las dificultades encontradas para entrar en el mercado de trabajo van a influir negativamente en la motivación y actitud de búsqueda de empleo y el compromiso futuro con el trabajo, de forma más acusada cuanto más se alargue el tiempo de entrada al mismo (7-9).

\section{- El diferente impacto del trabajo (remunerado) en hombres y mujeres}

Se han encontrado diferencias en el impacto del desempleo sobre el hombre y la mujer. Un estudio de la Agencia de Salut Publica de Barcelona (ASPB) con datos de la Encuesta de Salud de Cataluña de 2002 encontró que las mujeres catalanas en paro mantenían una mejor condición de salud que los varones desempleados y que la mujer trabajadora tenía un mayor riesgo de mala salud (mental) que la desempleada con subsidio (en proporción de 2 a1), justo lo contrario que el hombre (de 1 a 4). 
Se aporta como explicación que las mujeres suelen tener peores condiciones laborales que los hombres y una sobrecarga añadida por la "doble" jornada laboral - fuera y dentro del hogar - en muchos casos.

Por su parte, entre los varones, los trabajadores manuales, con hijos pequeños y sin subsidio, percibían de forma más dramática el incumplimiento de sus obligaciones de sustentar a la familia, con un riesgo añadido para su salud (mental) (10).

Consecuencias generales del desempleo y el trabajo precario (11).

Ni contigo $n i$ sin $t i$

Tienen mis males remedio

Contigo, porque me matas

$Y$ sin ti, porque me muero

COPLA Popular

La situación actual, caracterizada por un alto desempleo mantenido y el crecimiento del empleo precario, facilita la aparición de una forma de estrés laboral donde adquiere especial relevancia tanto la inseguridad del presente como el miedo al futuro (perder el trabajo, no encontrar uno nuevo), resultando tan estresante la preocupación por el riesgo de perder lo que se tiene como la pérdida en si, el despido, que abre la posibilidad de elaboración del duelo, al menos, por una pérdida real, el empleo.

Las consideraciones de Freud, en Duelo y melancolía (1915), nos pueden ayudar a entender el distinto impacto psicológico del despido (trauma puntual) frente a la percepción cotidiana (y continuada) de la amenaza del mismo, propia de las situaciones de inseguridad laboral (12).

Se han descrito múltiples riesgos del desempleo sobre la salud de las personas: aumento de la mortalidad general y por causa cardiovascular - con aumento de las tasas de hipertensión e hipercolesterolemia -, de las enfermedades crónicas, de la mortalidad en la infancia y de distintos padecimientos físicos (cefaleas y migrañas, enfermedad de Crohn). También se han descrito, junto a una percepción más negativa de la propia salud, el aumento de conductas de riesgo en relación con la duración del desempleo: tabaquismo, obesidad y sobrepeso, consumo de alcohol y otras drogas, sedentarismo y menos consumo de frutas y verduras $(1,13,14)$. Y en relación con la salud psíquica del sujeto, cuadros ansioso-depresivos de carácter reactivo, abuso de alcohol y otras sustancias, tabaquismo, distintos síntomas psicológicos y psicosomáticos en adultos jóvenes, trastornos del sueño, sentimientos de culpa, conflictos familiares, violencia doméstica e intentos de suicidio (15). 
PARA EL DEBATE

En el caso del empleo precario se suma a la inestabilidad presente e inseguridad en el futuro, el efecto directo de la baja consideración social, bajos sueldos, jornadas extenuantes, altos ritmos de trabajo, elevado estrés y exigencias, amenazas de despido, condiciones ambientales nocivas, alta exposición a situaciones peligrosas y bajo o nulo control de la persona sobre la tarea desempeñada, pudiendo afectar al bienestar (psíquico) de la persona aun más que la situación de desempleo subsidiado $(2,16)$.

Se ha asociado la precariedad laboral con un aumento del nerviosismo y la ansiedad, miedo, sufrimiento, depresión y pérdida de la sociabilidad y las relaciones de amistad (2). También con un mayor riesgo de fatiga crónica, de padecimientos crónico-degenerativos y cardio-vasculares (infarto, hipertensión y diabetes), de intoxicaciones, tumores y mayor siniestralidad laboral.

A pesar de que una persona desocupada puede empeorar su ya reducido bienestar (psíquico) al verse obligada a aceptar un trabajo precario e inadecuado para su capacitación profesional, esto no suele tenerse en cuenta al diseñar las políticas de empleo, situación que ha empeorado con las políticas de austeridad seguidas por los gobiernos en la crisis actual.

En España, más del $90 \%$ de las nuevas contrataciones son temporales y la proporción de nuevos contratos indefinidos disminuye continuamente, por lo que no es de esperar una reducción de la precariedad del mercado laboral ni de su impacto negativo sobre la salud. En palabras de L. Andor, Comisario de Empleo de la UE, "desafortunadamente, en 2014 no podemos decir que tener un trabajo equivalga a conseguir un estándar de vida decente".

DOS. Retos que plantean las crisis (financieras) sobre la salud (mental). Desempleo y protección socio-sanitaria

Las crisis económicas plantean retos a los gobiernos para proteger la salud de las personas acuciadas por la misma. En la crisis actual, Levy y Sidel (17) consideran que los gobiernos deben afrontar especialmente los siguientes retos por su impacto sobre la salud:

- El desempleo y el trabajo precario

- La desnutrición en niños, mayores, mujeres embarazadas y enfermos crónicos

- Las personas sin hogar o en infraviviendas

- La salud infantil

- Los trastornos mentales

- La mortalidad general de la población 
- La protección del medio ambiente

- La accesibilidad a los servicios públicos de salud

- La protección y cohesión social y los derechos de los trabajadores

La Oficina Regional para Europa de la OMS, en su informe de 2011 sobre el impacto de la crisis económica en la salud mental, también remarca al desempleo junto con el empobrecimiento, los problemas de alojamiento y la menor accesibilidad a los servicios públicos, como los principales factores de riesgo para la salud mental (60)

Los estudios de corte sociológico sobre el desempleo se han centrado en su impacto negativo sobre la calidad de vida y el nivel de satisfacción de las personas, su grado de "bienestar psíquico", por tanto. Sus conclusiones, sin embargo, no son directamente trasladables a la clínica psicopatológica, más constreñida a establecer un diagnóstico clínico y psicopatológico a partir de lo expresado por el paciente y su conducta $(1,4,10,13)$.

¿Qué impacto negativo se puede esperar del desempleo sobre la calidad de la vida de las personas?. Múltiple y de distinta índole: menos ingresos económicos, menos incentivos vitales, menos objetivos, actividades y posibilidades de tomar decisiones y desarrollar nuevos conocimientos y capacidades y un mayor riesgo de ver alterados, por último, el equilibrio psicológico y las relaciones sociofamiliares. Frente a lo anterior, el grado de protección socio-sanitaria de las personas puede actuar de contrapunto (18): el riesgo de padecer un problema psíquico va a ser 5 veces mayor en desempleados sin subsidio que en los activos. Y en los desempleados con subsidio la relación con los activos se reduce a 2 veces mayor.

El objetivo de reforzar la protección social y sanitaria en tiempos de crisis es doble: mantener la salud de la población y ayudar a la recuperación económica mediante un gasto social eficiente $(14,19)$.

\section{TRES. Desempleo, trabajo precario y salud (mental)}

\section{Principales riesgos para la salud (mental) asociados al desempleo}

La pérdida del empleo constituye uno de los principales factores de riesgo de aparición de problemas de salud mental tales como la ansiedad, el insomnio, la depresión y las conductas disociales y autolesivas.

Eisenberg y Lazarsfeld (1938) han establecido para los años de la Gran Depresión las consecuencias negativas para la salud asociadas al alto desempleo: deterioro emocional, descenso de la autoestima, pérdida del sentido estructurante del tiempo vital y apatía e indolencia, con disminución de las actividades cotidianas y de la participación social y política (20). 
PARA EL DEBATE

Resultados comparables encontramos en estudios de los años 70 en relación con la crisis del petróleo, lo que expresa el mantenimiento de una misma cultura laboral y la estabilidad de las respuestas de los trabajadores frente a este tipo de situaciones.

El desempleo puede causar, por sí mismo, la aparición de los primeros problemas de salud mental en una persona previamente sana, especialmente entre desempleados de más de 6 meses de duración, personas con educación superior y grupos minoritarios, apareciendo cuadros de tipo depresivo y de ansiedad generalizada que no habían padecido con anterioridad.

Efectos negativos del desempleo sobre la salud de la población.

Habiendo cumplido el crecimiento económico y social del siglo XX un importante papel en la disminución de las tasas de mortalidad y en el crecimiento de la esperanza de vida (21), estudios longitudinales sobre las fases expansivas y recesivas de estos grandes ciclos económicos han detectado que las cifras de mortalidad general tienden a aumentar más en las épocas de expansión que en las de recesión (22). La relación entre nivel de vida de una población, índices de mortalidad y tasas de suicidio difiere entre unos países y otros, en función de las características de su desarrollo económico y de su grado de protección socio-sanitario.

Así lo confirman Blasco-Fontecilla y al. (23). Mientras en los países con mayor desarrollo económico-social (UE, EEUU, Canadá, Nueva Zelanda y Australia) existe una correlación negativa desde hace años entre crecimiento de la riqueza nacional y tasa de suicidios (a mayor riqueza menos suicidios), países y regiones en desarrollo como América Latina y el Caribe, mantienen una tendencia al alza de la tasa de suicidios a pesar de crecer su economía, lo que se debe, a su juicio, a la carencia en estos países de las infraestructuras socio-sanitarias adecuadas y recursos suficientes para una buena atención de los problemas de salud mental que lleve consigo medidas eficaces en la prevención del suicidio en personas con trastornos mentales severos y, por tanto, con mayor riesgo de poder actuar contra su vida.

\section{Sobre las conductas suicidas}

La OCDE, en su Informe de 2013 y con carácter general, asocia tasas más altas de suicidio con la presencia de bajos ingresos, abuso del alcohol y drogas, estar desempleado o ser soltero $(24,25,26)$.

Existiendo otras variables, junto al desempleo, que afectan al riesgo de suicidio (pobreza, trastornos psíquicos, carencia de lazos sociales o cultura de la población frente a la muerte) (27), en los periodos de recesión económica se ha encontrado una alta correlación entre tasas de desempleo y de suicidio (28-31), especialmente entre varones de 30 a 49 años $(32-34)$ y jóvenes entre 16 y $24(35,36){ }^{1}$.

Los últimos datos sobre mortalidad en España suministrados por el INE para 2012 establecen el suicidio como la primera causa de muerte (17.8\%) en los varones entre los 25 y los 34 años. Y para la población entre 15 y 39 años, las causas externas (básicamente el suicidio y los accidentes de tráfico) constituyen la primera causa de muerte $(15.4 \%)$ (35). En consonancia, los datos oficiales existentes sobre el grado de ocupación, paro, empleo precario, desempleo de larga duración y tasa de NINIS entre los jóvenes españoles es espeluznante (36). 
Un ejemplo de lo anterior se ha podido ver en el gran impacto de la crisis asiática de finales de los 90 sobre el suicidio, que creció un 39\% el año 1998 en Japón respecto del año anterior, un 44\% en Hong-Kong, un 45\% en Corea del Sur y un $60 \%$ en Tailandia.

Podemos plantearnos, por tanto, que la tasa de suicidios, que ha venido disminuyendo en el continente europeo de manera constante en los últimos 20 años, podría estar viéndose afectada por la crisis económica y financiera actual y su impacto sobre el desempleo.

Stuckler y McKee han tratado de cuantificar en algunos países europeos la relación entre desempleo y tasa de suicidios en varones menores de 65 años: por cada punto de aumento del desempleo crecería un $0.8 \%$ la tasa de suicidios y para un crecimiento del paro superior al $3 \%$ se podría esperar un aumento de un $4.5 \%$ en la tasa de suicidios (28).

Shepherd y Barraclough relacionan el riesgo de suicidio también con estar desempleado, haber tenido cambios de trabajo en los últimos 3 años y presentar algún síntoma psiquiátrico (37). Y Charlton añade a la alta movilidad laboral, para Inglaterra y Gales, el residir en zonas rurales, las rupturas matrimoniales y el aislamiento social (34).

Mayor concreción de la relación entre el suicidio y las circunstancias económicas del país nos lo da Italia, donde al registrarse varios motivos de suicidio (enfermedad física, enfermedad mental, causas afectivas, causas de honor, motivos económi$\cos$ y no identificados) se puede valorar, junto a la tendencia general, las variaciones debidas a una causa específica - en este caso la económica -, y ver si ha crecido con la crisis económica. Pues bien, se ha encontrado un aumento de dicha causa entre 2007 y 2010 coincidiendo con el aumento de la tasa de desempleo, mientras que los índices de suicidio atribuibles a las demás causas permanecían estacionarios (19).

El Informe Eures sobre el suicidio en Italia con datos del Instituto Nacional de Estadística encuentra un crecimiento de la tasa general de suicidios entre 2008 y 2010 de un $7.8 \%$ que rompe la tendencia decreciente de años anteriores (38). El alto crecimiento relativo del número de suicidios por razones económicas afecta casi exclusivamente a los varones y tiene un impacto pequeño sobre el resultado global, al estar muy por debajo en número de los casos de suicidio por enfermedad mental, el motivo más alto registrado. El crecimiento de suicidios en varones es extensible también a los intentos de suicidio, rompiendo una tendencia histórica de distribución en favor de la mujer. Entre los varones, por último, son los desempleados los de mayor riesgo, seguidos de los pequeños empresarios y trabajadores autónomos, lo que indica un crecimiento del número de suicidios en consonancia con el aumento de la tasa de desempleo.

Tasas de suicidio y nivel de protección social

Según Martin McKee, mantener un alto nivel de protección social en los periodos de recesión mitiga su impacto sobre la tasa de suicidios. En el caso de la 
PARA EL DEBATE

UE, de hecho, uno de los predictores más fuertes de las tasas de suicidio es el porcentaje de gasto público sanitario respecto del total (39). En España se produjo un aumento del índice de suicidios en los años 80 coincidiendo con una gran subida del paro, algo que no ocurrió en Suecia en la crisis de los 90, donde el desempleo no tuvo un impacto significativo sobre la tasa de suicidios por el enérgico papel de los programas de ayuda y apoyo social desarrollados por el estado sueco $(40)^{2}$.

En la crisis actual del continente europeo, los tres países con mayor subida de la tasa de suicidios registrada entre 2008 y 2009 - Letonia, Irlanda y Grecia - habían llevado a cabo las mayores medidas de austeridad económica y habían reducido de forma severa el nivel de protección social de la población (28).

El informe referido de la OCDE sobre salud, confirma en estudios previos a la crisis actual la relación existente entre condiciones económicas adversas, desempleo y mayores tasas de suicidio (41-43). Dicho Informe constata un aumento inicial de las tasas de suicidio en países especialmente castigados por la crisis económica aunque considera no demostrada la continuidad de dicha tendencia en años sucesivos. En el caso de Grecia - donde aumentó la tasa de mortalidad por suicidio entre 2007 y 2009 - dicha tasa se habría estabilizado de 2009 a 2010 a pesar de seguir empeorando las condiciones económicas de la población: al pico inicial de la tasa de suicidios por el estallido de la crisis, habría seguido una progresiva estabilización por la adaptación de la población a la nueva situación ${ }^{3}$. Por contra, diversos estudios sobre la situación de Grecia han mostrado un crecimiento general de la tasa de suicidios de 2.8 a 6/100.000 habitantes desde el inicio de la crisis, y eso a pesar del cuestionamiento de dichas cifras oficiales por distintos investigadores, al estar socialmente estigmatizado el suicidio en Grecia por la Iglesia Ortodoxa.

En todo caso, Grecia se ha convertido en el paradigma dentro de la UE de las consecuencias negativas de las políticas de recorte generalizado sobre el bienestar de la población. Aparte del crecimiento de la tasa de suicidios en varones del $22.7 \%$ entre 2007 y 2009, se duplicaron los casos de depresión entre 2008 y 2009 con un deterioro generalizado posterior de los índices de trastornos mentales y abuso de sustancias $(44,45)$. Y si consideramos el impacto sobre la salud pública, la reducción de los presupuestos para sanidad en un $23.7 \%$ entre 2009 y 2011 han deteriorado los recursos sanitarios públicos asistenciales y de carácter preventivo hasta el punto de rebrotar enfermedades infecciosas como la malaria y multiplicarse los nuevos casos de VIH entre los consumidores de drogas inyectables.

\footnotetext{
Los fondos para los desempleados en ambos países eran muy diferentes: en cantidad total la relación era de

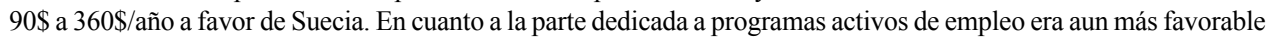
a Suecia: un $75 \%$ frente a un $10 \%$.

Un dato indirecto en sentido contrario sería el gran crecimiento habido durante 2011 - en consonancia con el aumento del desempleo registrado - de las llamadas de búsqueda de socorro al teléfono de la esperanza, más del doble que el año anterior.
} 
Uno de los pocos estudios sobre la relación entre crisis financiera y tasas de suicidio en España realizado con los datos oficiales de 2005 a 2010 mediante el análisis de series temporales interrumpidas, encuentra un aumento relativo del $8 \%$ respecto de la tendencia predecible para estos años de no haber mediado la crisis financiera de comienzos del 2008, siendo más relevante en varones de 40 a 64 años y en el norte y este del país frente a la zona centro. El índice de muertes por accidentes fortuitos usado como control no varió en estos años. A pesar de no disponer de datos posteriores al 2010, sugieren la existencia de un nuevo repunte en la $2^{\mathrm{a}}$ fase de esta larga crisis ${ }^{4}(46,47)$.

\section{Sobre las tasas de mortalidad}

Estudios sobre las distintas fases de los ciclos económicos han observado que el impacto sobre la salud en las fases de recesión económica y de expansión no coincide siempre con lo que se podría prever de forma intuitiva (22).

Un ejemplo ha sido la disminución de la mortalidad global en Estados Unidos durante la Gran Depresión en un 10\% para 1931. Aunque la tasa de suicidios consumados aumentó entre 1929 y 1932 un 16\%, se vio compensada por el gran descenso habido en las muertes por accidentes de tráfico. Dicha tasa de suicidios variaba en cada Estado según el grado de aplicación de los programas del New Deal y el número de bancos que habían ido a la quiebra. La menor renta disponible de las familias y los individuos redujo el consumo de alcohol y nicotina y el uso del automóvil, con un doble beneficio: la reducción del tráfico rodado (y de accidentes mortales) y de la vida sedentaria de los americanos.

Este descenso de la mortalidad global por "motivos económicos" estaba reforzado por el apoyo a la salud pública promovido por el New Deal, centrado en la atención sanitaria y la salud mental, las ayudas en alimentos y para vivienda, con resultados más tangibles en los Estados que más habían desarrollado estos programas, con mejores datos relativos en enfermedades infecciosas, mortalidad infantil y suicidios (19). El análisis pormenorizado permitió descubrir el empeoramiento de otros indicadores aparte del suicidio, como era el caso de las muertes violentas por homicidio.

En su estudio sobre condiciones económicas y salud en Estados Unidos entre 1972 y 1991, Ruhm encuentra también una tasa de mortalidad total menor durante los periodos de recesión en 9 de 10 causas de muerte, aumentando sólo la tasa de suicidios (48).

En la crisis actual Islandia también ha mejorado ligeramente sus tasas de mortalidad entre 2007 y 2009: la reducción generalizada de la jornada laboral ha amorti-

\footnotetext{
4 En Cataluña su Consejero de Salud, al presentar recientemente un Programa para la Prevención del Suicidio, ha informado del crecimiento de la tasa de suicidios (consumados) entre 2007 y 2011 en un 25.9\% (desde el mínimo histórico de un 5.18/100.000 hab. de 2007 hasta un 6.52\% en 2011) (47).
} 
PARA EL DEBATE

guado el número de desempleados y aumentado el tiempo disponible para el ocio y el descanso nocturno. Al disponer de menos ingresos se ha reducido el consumo de alcohol y nicotina y recuperado el gusto por el pescado local, más sano y barato, frente a la moda por la comida rápida de los últimos años.

En cuanto a los datos de mortalidad en nuestro país, es inquietante la cifra relativa al crecimiento en un 58\% entre 2007 y 2010 de las tasas de mortalidad materna (TMM) (19). Al tratarse de un indicador sanitario con grandes fluctuaciones anuales debe confirmarse esta tendencia en años sucesivos y valorar los datos en el contexto de los cambios sanitarios y de otro orden ocurridos en nuestro país.

Partimos de una tasa de mortalidad materna muy baja en el contexto europeo (6/ 100.000 para 2008) y, junto con Italia, la mayor prevalencia de nacimientos en mujeres de más de 35 años de toda Europa. Entre 1996 y 2005 se venía constatando un cambio de tendencia con un crecimiento de la mortalidad en un $20 \%$ por el aumento generalizado en la edad de maternidad, especialmente entre mujeres sin nacionalidad española, lo que podría avalar el papel de la inmigración en el incremento de las TMM (49).

Junto al crecimiento de las técnicas de reproducción asistida en los últimos 10 años conviene valorar el impacto de las políticas de ajuste de estos años sobre el nivel de protección sanitaria de esta población: ha disminuído la accesibilidad al sistema sanitario público con especial afectación a la población inmigrante, ha aumentado la aportación económica directa de los beneficiarios en materia de medicamentos y se ha reducido la calidad asistencial en base a los recortes en medios y personal, con mayores tiempos de espera y menos tiempo de atención y seguimiento.

Principales consecuencias del desempleo (y el trabajo precario) sobre la salud (mental) de las personas (50).

Distintos estudios de orden clínico hacen hincapié en el incremento de las reacciones desadaptativas (Duelo), junto a los trastornos mentales comunes (ansiedad y depresión), las recaídas en personas con trastornos mentales previos y el aumento del consumo de sustancias y de la aparición de conductas disociales.

Al situar el problema principalmente en el campo de las reacciones adaptativas y de los procesos de elaboración del duelo, disponemos de una visión menos dramática (y más realista) de estos problemas en su perspectiva médico-sanitaria. Ante la pérdida de empleo las personas ponen en marcha mecanismos de adaptación no necesariamente patológicos, pudiendo aparecer temporalmente síntomas de ansiedad, estrés o depresión, síntomas con cierta tendencia a la cronificación pero que rara vez 
desencadenan complicaciones psiquiátricas graves, como es el caso de la depresión mayor y las conductas suicidas. Cuando hay respuestas psicopatológicas severas debemos investigar y descartar siempre la presencia de un trastorno psiquiátrico previo, incluyendo las alteraciones de la personalidad con baja tolerancia a la frustración.

Desde el campo psicoanalítico se ha contemplado la tarea del duelo desencadenado por la pérdida del trabajo como un proceso gradual hacia la recuperación psíquica mediante pasos sucesivos: examen de la realidad, clausura, sobreinvestidura y desasimiento (12).

Kubler-Ross, por su parte, ha ordenado el proceso de elaboración del duelo por la pérdida del empleo en cuatro estadíos o periodos evolutivos: de negación del problema, de sentimientos generalizados de ira, periodo depresivo y, por último, de aceptación de la pérdida y de afrontamiento de la nueva situación (51) ${ }^{5}$.

Si consideramos el proceso de elaboración del duelo en parados de larga duración $(4,13)$, a un rápido deterioro de su salud percibida, tanto física como mental, entre los 3 y los 6 primeros meses por la pérdida brusca de distintos aportes psicosociales (disponibilidad económica, seguridad física, relaciones laborales, pérdida de estatus, etc.), le sigue un proceso de estabilización de la situación entre los 7 y los 12 meses. Desde los 12 meses hasta los 24 se entra en una fase de mayor acomodación con pocas variaciones respecto al periodo anterior (52).

El estudio de Astell-Burt y Feng (53) sobre el impacto de la crisis actual en el Reino Unido constata un aumento de la mala salud de la población especialmente entre los desempleados al comienzo del 2008, seguida de una reducción de esta tendencia en la segunda mitad del 2008 y en 2009. Dos años después, sin embargo, los indicadores de mala salud han rebrotado de forma generalizada, afectando ahora especialmente a los que, habiendo mantenido su empleo hasta entonces, habían sufrido condiciones crecientes de estrés en el trabajo, por la inseguridad del mismo, su precarización y el temor cada vez más real a perderlo y ver reducidos sus ingresos, condiciones y expectativas vitales (54).

Relaciones entre desempleo, malestar y uso de servicios sanitarios

\section{Uso de servicios sanitarios}

La recesión económica - con su correlato de estrés laboral y desempleo -, al aumentar el malestar de la población afectada tiende a generar una mayor demanda

\footnotetext{
$5 \quad$ Se trata de una adaptación realizada en 1987 para el desempleo de las etapas descritas previamente para las enfermedades terminales en su publicación, ya clásica, de 1969.
} 
PARA EL DEBATE

de atención sanitaria, al buscar en el médico de atención primaria alivio a los problemas de estrés, ansiedad y depresión reactiva. También pueden verse afectadas las derivaciones a los CSM y la prescripción y consumo de antidepresivos y otros medicamentos psicotropos.

Sin embargo, el uso mayor o menor de servicios sanitarios sigue siendo un tema controvertido, al estar afectada la demanda asistencial por distintos factores ${ }^{6}$. Desde sectores profesionales se señala un aumento de las consultas médicas, del consumo de medicamentos y de las hospitalizaciones especialmente entre las personas desempleadas, por sus problemas de ansiedad, depresión, alcoholismo y consumo de drogas (55).

En una evaluación de primeras consultas llevada a cabo entre mayo y septiembre de 2013 en el CSM de Hortaleza (Madrid), casi la mitad de los pacientes achacan su malestar psíquico a "consecuencias de la crisis" siendo este el principal motivo de derivación al centro para tratamiento (56).

Y en un estudio realizado entre 2006 y 2010 en la red de atención primaria se vio un aumento entre el 29 y el $48 \%$ del número de consultas al MAP por síntomas agudos de depresión, ansiedad y consumo de alcohol, siendo la pérdida reciente de empleo el predictor más consistente de dicho crecimiento, agravado en su caso, si había otro miembro de la familia desempleado y si tenían dificultades para hacerse cargo de obligaciones básicas del hogar, como el pago de la hipoteca (50).

En sentido contrario, en un estudio llevado a cabo en La Rioja, aumentó el uso de servicios informales de salud (sanadores, medicina alternativa, curanderos, masajistas, fisioterapeutas y otros) pero no las visitas médicas, lo que se achaca a las características culturales de dicha zona (52).

Un estudio realizado en Filadelfia entre 2005 y 2008 con personas amenazadas de embargo - una de las consecuencias más violentas de la crisis - ha encontrado un incremento en las visitas a urgencias y en las hospitalizaciones por descompensación de la hipertensión arterial o complicaciones renales de la diabetes especialmente entre personas menores de 50 años, relacionándolo con causas económicas: se trata de personas sin ingresos suficientes que han de priorizar el pago de la vivienda a costa de los cuidados necesarios para el control de su enfermedad crónica. El índice de complicaciones aumentaba cuando el riesgo a quedarse sin vivienda se hacía más inminente (19).

En Grecia, por último, la población ha suspendido sus pólizas de seguro privado por falta de ingresos y aumentado el uso de los servicios sanitarios públicos tanto primarios (un 21.9\%) como hospitalarios (un 6.2\%) (44) .

\footnotetext{
6 Un importante mediador de la demanda, la accesibilidad a los servicios, se ha visto reducida estos años en todo el territorio nacional por la exclusión de derechos a la asistencia sanitaria de una parte de la población, aumento del coste directo de la atención médico-farmacéutica, reducción de los recursos sanitarios públicos y crecimiento de los tiempos de espera.
} 
PARA EL DEBATE

\section{Prescripción y uso de medicamentos}

La prescripción y uso de medicamentos ha crecido de forma variable en toda Europa. Entre 2007 y 2009 aumentaron en España un 17\% las recetas de antidepresivos. Comparativamente, en el RU crecieron un $22 \%$ mientras que en Suecia, un país con medidas más eficaces sobre el desempleo, sólo creció el consumo un 6\% (19).

Es difícil valorar, sin embargo, el impacto de la crisis actual sobre el incremento del consumo de antidepresivos y ansiolíticos.

Un crecimiento significativo en la prescripción y uso de antidepresivos, ansiolíticos e hipnosedantes en España (y en Europa) empieza ya con el lanzamiento del antidepresivo de Lilly conocido como Prozac (fluoxetina) a finales de los 80 . En los 90 y hasta la actualidad ha crecido la prescripción de estos medicamentos especialmente en la red de atención primaria, sin que guarde relación con el aumento de la prevalencia de trastornos depresivos en población general establecida mediante estudios epidemiológicos fiables (54).

$\mathrm{Al}$ crecimiento de los problemas de salud mental en la crisis, hay que sumar la llegada de una nueva familia de antidepresivos más fáciles de seguir por los médicos generales que los "viejos" antidepresivos y mejor aceptados por los pacientes junto a la banalización del diagnóstico de depresión, ampliado dentro de la propia red asistencial a distintos malestares emocionales desencadenados por las dificultades del vivir (57). Y por último, la ampliación del uso de los nuevos antidepresivos en enfermedades médicas de curso crónico que se acompañan en algún momento de su evolución de algún tipo de malestar psíquico.

CUATRO. La gestión de las crisis. Medidas para mitigar su impacto negativo sobre la salud (mental) de la población

\footnotetext{
"Primun non nocere" (Lo primero, no hacer daño) MÁxima ATRIBUídA A Hipócrates (460 a 370 a.C.)
}

Ni la peor catástrofe económica repercute necesariamente en un daño a la salud de la población si los gobiernos toman las medidas adecuadas y suficientes para protegerla (19).

La Oficina Regional para Europa de la OMS en su informe de 2011 sobre el impacto de la crisis económica en la salud mental propone desarrollar programas activos de empleo, dar apoyo a las familias con dificultades económicas, reducir la disponibilidad del consumo de alcohol y potenciar los servicios básicos de salud (y salud mental) (58). 
PARA EL DEBATE

Junto a los programas de empleo y la mejor accesibilidad a los servicios de atención primaria y salud mental, dan prioridad a los programas de apoyo psicológico orientados hacia la prevención y detección precoz de los problemas de salud y centrados en intervenciones psico-educativas y psicoterapéuticas breves, eficaces y adaptadas a las necesidades de la población.

\section{Medidas generales de carácter institucional}

La primera obligación de los gobiernos democráticos en estas situaciones ha de ser arbitrar fórmulas para proteger eficazmente la salud de la población y evaluar de forma objetiva y transparente el impacto de las políticas emprendidas frente a la crisis sobre su bienestar.

Reforzar la protección socio-sanitaria de la población, especialmente de los grupos de riesgo, mitiga el impacto de la crisis - especialmente la relación entre desempleo y suicidio -, al mantener un alto grado de cohesión social. Los puntos fuertes son el mantenimiento de una atención sanitaria universal, ayudas a la vivienda, subsidios para los desempleados y pensiones suficientes, cuatro aspectos que afectan de manera directa a las personas más sensibles a la crisis: los enfermos, los pobres y los sin techo, los parados y las personas mayores (40).

Un reciente estudio ha cuestionado los recortes presupuestarios, cambios normativos y organizativos llevados a cabo en España por los gobiernos central y autonómicos en materia de sanidad y bienestar social, por su impacto negativo directo sobre el cuidado de la salud y el riesgo de un desmantelamiento progresivo de nuestro Sistema Nacional de Salud que dañaría en el medio y largo plazo aun más la salud de la población. Helena Legido-Quigley denuncia además la falta de indicadores de salud y de datos oficiales fiables sobre distintos aspectos de la sanidad española: "No hay datos, es algo terrible. Las cifras oficiales se publican hasta con 3 y 4 años de retraso" ". Con los pocos datos existentes y en base a indicadores indirectos piensa que están creciendo en España los cuadros depresivos y ansiosos y los suicidios en relación con la crisis y su gestión $(35,45)^{8}$.

Contar con redes sociales sólidas - familia, amigos, etc. -, constituye un soporte psico-físico natural de primer orden frente a las crisis y los efectos negativos del desempleo, hasta el punto que el grado de apoyo social (real y percibido) es un buen predictor del riesgo de aparición de trastornos físicos y emocionales en las personas desempleadas.

\footnotetext{
El déficit de información de la sanidad española es difícil de entender sin un cierto desorden de coordinación entre estado central y comunidades autónomas y el mantenimiento de restos de una cultura política que prefiere ocultar la realidad sanitaria en favor de su uso propagandístico.

8 Los datos sobre defunciones según la causa de muerte para 2012 presentados por el INE en nota de prensa de 31.1.2014 podrían ir en la misma dirección: en relación al 2011 ha crecido en un $12.2 \%$ la mortalidad por trastornos mentales y del comportamiento (que incluyen las demencias seniles y vasculares) y un $11.3 \%$ el número de suicidios - un 77\% corresponde a varones -, con una tasa de 7.6/ 100.000 hab., la más alta desde el año 2005 (35).
} 
Medidas específicas de carácter socio-sanitario y psico-social.

Desgraciadamente, pocas medidas eficaces se han podido establecer de manera exclusiva desde el sistema sanitario y la red de salud mental $(59,60,61,62)$.

La medicina primaria debe jugar un papel importante de contención y ayuda a los pacientes en situación de desempleo mediante la instauración de medidas de educación para la salud, la implantación y mantenimiento de prácticas saludables y el cribaje de los efectos más negativos que dicha situación puede generar sobre su salud física y psíquica (63).

Desde la psicología, los programas de apoyo psicológico y laboral bien diseñados y correctamente financiados han demostrado también su utilidad.

En el ámbito de la psicología clínica, medidas de apoyo psicoterapéutico breve pueden aumentar la resistencia psíquica de los sujetos en situaciones de duelo por la pérdida del trabajo y ayudar a otras personas de riesgo (sujetos con bajos ingresos, jóvenes, familias monoparentales, minorías étnicas, inmigrantes, ancianos, enfermos mentales y personas sin techo) a afrontar y resolver mejor las dificultades añadidas por esta situación.

Desde la psicología laboral, enseñar al desempleado nuevas habilidades de carácter relacional que mejoren su capacidad organizativa, actitud y respuesta frente a las entrevistas de empleo, puede aumentar su nivel de "empleabilidad".

En los casos más graves, marcados por la presencia de elementos de violencia laboral, se va a hacer necesario un seguimiento psicoterapéutico reglado, prolongado y sistemático (64).

Medidas de carácter laboral: Políticas activas de empleo y de reciclaje profesional

Tener un empleo estable y adecuado a las competencias y características del trabajador es la mejor medicina que el Estado puede ofrecer a los ciudadanos.

En situaciones de alto nivel de desempleo es prioritario ayudar a la gente a encontrar trabajo mediante políticas activas de empleo eficaces que incluyan junto al apoyo emocional y psicosocial, información laboral, formación y reciclaje profesional.

Ejemplo de referencia es Suecia. Junto al subsidio de desempleo ha desarrollado programas de búsqueda activa de trabajo y aumento de la resistencia psíquica de las personas ante la pérdida del mismo. Desde que la persona queda en paro, un asesor laboral le ofrece apoyo individualizado, su conocimiento y experiencia y un plan específico y viable de vuelta al trabajo. Contar con programas personalizados de empleo disminuye el riesgo de aparición de cuadros depresivos secundarios a la pérdida de trabajo o al riesgo de perderlo (19).

Ejemplo de gestión eficiente de estos programas es Dinamarca, donde los beneficios económicos generados por el aumento de la productividad laboral y la reducción de la dependencia del sistema de protección social han sido superiores a los gastos generados en su implantación y desarrollo. 
PARA EL DEBATE

Estos países, junto con otros como Alemania y Holanda, priorizan itinerarios individualizados que implican y comprometen a los desempleados de forma que, tras el periodo de formación y ante una oferta de trabajo adecuada a su capacitación, no aceptarla puede llevar consigo la pérdida del subsidio.

Nuestro país, dentro de la UE, deja mucho que desear en cuanto al diseño y eficacia en la implantación de políticas activas de empleo: más de la mitad de nuestros parados lleva más de un año buscándolo sin éxito. Es notorio el fracaso del INEM en esta tarea y no es de extrañar que la modernización de los servicios públicos de empleo continúe siendo una petición habitual y sistemática de la Comisión Europea al gobierno español.

Nuestro mercado de trabajo tiene problemas crónicos desde el comienzo de la transición a la democracia que no han sido suficientemente resueltos. A mediados de los años 70, el efecto combinado de crisis económicas sucesivas, el retorno de alrededor de un millón de trabajadores españoles desde Alemania y otros países europeos y el enorme aflujo de generaciones jóvenes de hombres y mujeres al mercado laboral hizo que el desempleo alcanzara cifras muy elevadas, afectando en las décadas de los años ochenta y noventa a cerca de una cuarta parte de la población activa: la tasa de desempleo de $24.2 \%$ alcanzada en 1994 es comparable a la de los años de la Gran Depresión americana del 29 (65).

Frente a la importancia de las medidas activas señaladas en favor del empleo para el mantenimiento de la salud (mental), las políticas de austeridad a ultranza siguen facilitando su destrucción. La experiencia de nuestro país hasta la fecha así lo confirma. ${ }^{9}$

\section{BIBLIOGRAFÍA:}

(1) Jahoda M. Empleo y desempleo: un análisis sociopsicológico. Madrid: Morata, 1987.

(2) Amable M, Benach J, Benavides FG, Menedez M, Porthé V. ¿Cuáles son los efectos de la precariedad laboral sobre la salud mental?. Resultados exploratorios en trabajadores temporales. En Amable M. La precariedad laboral y su impacto en la salud. Un estudio en trabajadores asalariados en España. Tesis doctoral. Barcelona: Universitat Pompeu Fabra, 2006.

(3) Coriat B. Los desafíos de la competitividad. Buenos Aires: Piette- Conicet, 1994.

(4) Alvaro JL. Desempleo y bienestar psicológico. Madrid: Siglo XXI, 1992.

(5) Barrio F, Echevarría A, Martínez B y Txurimendi JM. Instrumentos para medir factores psicosociales y síntomas ansiosos y depresivos. En: Páez D et al. Salud Mental y factores Psicosociales. Madrid: Ed. Fundamentos, 1986; 184-213.

(6) Garrido A. Desempleo. En: Reyes R (Dir): Diccionario crítico de Ciencias Sociales.

\footnotetext{
$9 \quad$ Al comienzo del 2014, seis años después del comienzo de la crisis en España, la tasa de desempleo es de un $26.03 \%$, sólo superada en la zona euro por Grecia.
} 
Madrid y Mexico: Plaza y Valdes, 2009.

(7) Garrido Luque A. Consecuencias psicosociales de las transiciones de los jóvenes a la vida activa. Madrid: Editorial Complutense, 1992.

(8) Garrido Luque A y Alvaro JL. La autoestima como reflejo de las transiciones de los jóvenes al mundo laboral. Rev. Interacción Social 1992; 2: 127-139.

(9) Torregrosa JR. Juventud, desempleo y proceso de socialización. Japan:The United Nations University, 1981.

(10) Paul KI, Moser K. Unemployment impairs mental health: Meta-analyses. Journal of Vocational behavior 2009;74: 264-282. 1990.

(11) Blanch JM. Del viejo al nuevo paro. Un análisis psicológico y social. Barcelona: PPU,

(12) Freud S. Duelo y melancolía. 1915. Obras completas. Tomo XIV. Buenos Aires: Amorrortu editores, 1992.

(13) Seva Díaz A, Molina Abril J A, García I. Desempleo y salud mental en España. Cuadernos aragoneses de economía 2002;12; 2: 292-306.

(14) Del Llano J y San Martín R. Desempleo y salud: Revisión y análisis crítico de los diferentes diseños de investigación utilizados. Revisiones en Salud Pública 1994; 3: 215-242.

(15) FADSP. Los efectos del desempleo sobre la salud. Madrid: Febrero del 2012.

(16) Butterworth $P$ et al. The psychosocial quality of work determines whether employment has benefits for mental health: results from a longitudinal national household panel survey. Occup Environ Med 2010; doi: 10; 1136/oem; 059030.

(17) Levy B S. y Sidel V W. Crisis económica y salud pública. Editorial. Medicina Social 2009; 4;1:92-98.

(18) Karanikolos M, Rechel B, Stuckler D, McKee M. Financial crisis, austerity, and health in Europe - Authors' reply. The Lancet 2013;382 Issue 9890:392.

(19) Stuckler D, Basu S. Por qué la austeridad mata. El coste humano de las políticas de recorte. Madrid: Taurus Pensamiento, 2013.

(20) Eisenberg P y Lazarsfeld P F. The Psychological effects of unemployment. Psychological Bulletin 1938; 35: 358-390.

(21) Brenner H A. Commentary: Ewconomic growth is the basis of mortality rate decline in the 20th century - experience of the United States 1901-2000. International Journal of Epidemiology 2005; 34: 1214-1221.

(22) Tapia Granados Jose A., La mejora de la salud durante las crisis económicas: un fenómeno contraintuitivo. Papeles de relaciones ecosociales y cambio global 2011; 113:121-137.

(23) Blasco-Fontecilla H, Perez-Rodriguez, M M, Garcia-Nieto R et al. Worldwide impact of economic cycles on suicide trends over 3 decades: differences according to level of development. A mixed effect model study.. BMJ Open 2012; 2:e 000785.

(24) Informe OCDE. Health at a Glance 2013: OECD Indicators.

(25) Crump $\mathrm{C}$ et al. Sociodemographic, psychiatric and somatic risk factors for suicide: a Swedish national cohort study. Psychological Medicine First 2013;1-11. DOI: 10.1017/ S0033291713000810.

(26) Qin P, Agerbo E, Mortensen PB. Suicide Risk in Relation to Socioeconomic, Demographic, Psychiatric, and Familial Factors: A National Register-based Study of All Suicides in Denmark, 
PARA EL DEBATE

1981-1997. American Journal of Psychiatry 2003; 160 (4): 765-772.

(27) Durkheim E. El suicidio. Bs. As: Ed.Losada, 1897.

(28) Stuckler D, Basu S, Suhrcke M, Coutts A, McKee M. The public health impact of economic crises and alternative policy responses in Europe: An empirical analysis. Lancet 2009; 374 (9686): 315-23.

(29) Orellano M. Desempleo y salud mental: Reflexiones en torno a la posible relación entre la desocupación y la conducta suicida. El caso Rosario. Comunicación libre al $6^{\circ}$ Congreso Nacional de Estudios del trabajo: Los trabajadores y el trabajo en la crisis. Bs. As, 13-16 de agosto del 2003.

(30) Crombie I K. Trends in Suicide and unemployment in Scotland, 1976-86. Brit.Med. Jour. 1989; 298: 782-84.

(31) Pritchard C. Is there a link between suicide and unemployment? Comparison of the UK with other European community countries. Brit Jour of Psichiatry. 1992; 160: 750-756.

(32) Platt $\mathrm{S}$ y Kreitman N. Long term trends in parasuicide and unemployment in Edinburgh, 1968-87. Social Psychiatry and Psychiatric Epidemiology 1990; 25, (1):56-61, DOI: 10. 1007/ BF 00789071.

(33) Platt S. Parasuicide and unemployment. British Journal of Psychiatry 1986; 149: 401405.

(34) Charlton J, Kelly S, Dunnell K. Trends in suicide death in England and Wales. Population Trends 1992;69:10-6.

(35) INE. Defunciones según la causa de muerte. Año 2012. Nota de prensa de 31.1.2014.

(36) Estrategia de emprendimiento y empleo joven 2013-2016. Ministerio de Empleo y Seguridad Social. Madrid 2013.

(37) Shepherd DM, Barraclough BM. Work and suicide: an empirical investigation. Brit. Jour. Of Psychiat. 1980;136: 469-478.

(38) Piacenti F. Il suicidio in Italia al tempo della crisis. II Rapporto Eures. Abril 2012.

(39) Innamorati $\mathrm{M}$, Tamburello $\mathrm{A}$, Lester $\mathrm{D}$ et al. Inequalities in suicide rates in the European Union's elderly: trends and impact of macroeconomic factors between 1980 and 2006. Can J. Psychiatry 2010; 55:229-38.

(40) McKee M, Stuckler D. The assault on universalism: how to destroy the welfare state. BMJ. 2011; 343:d7973. doi: 10.1136/bmj. d7973.

(41) Ceccherini-Nelli A, Priebe S. Economic Factors and Suicide Rates: Associations over Time in Four Countries Social Psychiatry and Psychiatric Epidemiology 2011; 46, (10):975-982.

(42) Classen TJ, Dunn R A. The Effect of Job Loss and Unemployment Duration on Suicide Risk in the United States: A New Look Using Mass-Layoffs and Unemployment Duration. Health Economics 2012; 21 (3):338-350.

(43) Zivin K, Paczkowski M, Galea S. Economic Downturns and Population Mental Health: Research Findings, Gaps, Challenges and Priorities, Psychological Medicine 2011; 41(07):13431348.

(44) Kondilis E, Giannakopoulos S, Gavana M, Ierodiakonou I, Waitzkin H, Benos A. Economic crisis, restrictive policies, and the population's health and health care: the Greek case. Am J Public Health 2013; 103(6):973-9.

(45) Legido-Quigley H, Otero L, Parra Dl, Alvarez-Dardet C, Martin-Moreno JM, McKee M. Will austerity cuts dismantle the Spanish healthcare system? BMJ 2013; 346:f2363. 
(46) Lopez Bernal JA, Gasparrini A, Artundo CM, McKee M. The effect of the late 2000s financial crisis on suicides in Spain: an interrupted time-series analysis. Eur J Public Health. 2013; 23(5):732-6.

(47) Europa Press: 17 de diciembre de 2013.

(48) Ruhm C J. Are recessions good for your health?. University of North Carolina at Greensboro, National Bureau of economic research (NBER), and The Institute for the Study of Labor (IZA), 1996.

(49) Luque Fernandez M A et al. Increase in maternal mortality associated with change in the reproductive pattern in Spain: 1996-2005. Journal of Epidemiology and Community Health 2009;63:433-438.

(50) Gili M, Roca M, Basu S, McKee M, Stuckler D. The mental health risks of economic crisis in Spain: evidence from primary care centres, 2006 and 2010. Eur J Public Health 2012;23(1):103-8.

(51) Kübler-Ross Elisabeth. On death and Dyng. New York: Macmillan, 1969.

(52) Pozo J A, Ruiz M A, Pardo A, San Martin R. Efectos de la duración del desempleo entre los desempleados. Psicothema 2002;14 (2):440-443.

(53) Astell-Burt T, Feng X. Health and the 2008 economic recession: evidence from the United Kingdom. PLoS One. 2013; 8(2):e56674. doi: 0.1371/journal.pone.0056674.

(54) Espino A. Trabajo, estrés y salud. En: Espino A coord, Vida laboral, estrés y salud mental. Madrid: AEN. Colección Estudios/ 49;17-39.

(55) Raventos S. Crisis económica y salud mental. Sin Permiso 28.2.2010. Revista electrónica semanal.

(56) Ceverino Dominguez A. A solas con la pulsión de muerte. Diez pantallazos sobre la relación entre exclusión y salud mental. Boletin de la AMSM 2013;36: 24-29.

(57) Espino Granado A coord. Presentación. En: Vida laboral, estrés y salud mental. Madrid. AEN. Estudios 49. 2012.

(58) OMS. Oficina Regional para Europa. 2011. Impact of economic crises on mental heath.

(59) Costa M, López E. Salud Comunitaria. Barcelona: Ediciones Martínez Roca, 1986.

(60) Libro verde sobre la salud mental. Comisión de Comunidades Europeas. Bruselas. COM 2005: 484 a final.

(61) Igartua J, Iraurgi J, Basabe N, Páez D y Celorio MJ. Enfermedad mental: grupos de autoayuda e integración social. Vitoria: Gobierno Vasco, 1994.

(62) Kaplan H et al. Sinopsis de Psiquiatría: Psiquiatría clínica y Ciencias de la Conducta. Buenos Aires: Panamericana. 7ma. Edición, 1997.

(63) Jin RL, Shah CP, Svoboda TJ. The impact of Unemployment on Health. A Review of the evidence. Journal of Public Health Policy 1997; 18 (3):275-301.

(64) Escudero A y Suarez V. El acoso psicológico en el trabajo: una forma severa de violencia interpersonal. En: Espino A coord. Vida laboral, estrés y salud mental. Madrid: AEN. Colección estudios/49, 2012: 119-140.

(65) Tapia Granados Jose A. Salud, economía y libertad: 40 años de crecimiento económico, transición política y condiciones de salud en España. Medicina Clinica (Barc) 2007; 128 (112): 463-7. 